\title{
Separação de Fases Induzida por Meio de Reação Química no Sistema Éter Diglicidílico do Bisfenol A e Piperidina com Poli(metacrilato de metila)
}

\author{
Filiberto González Garcia, Bluma G. Soares
}

IMA, UFRJ

\begin{abstract}
Resumo: O comportamento da separação de fases e da gelificação do sistema epoxídico, constituído pelo éter diglicidílico do bisfenol A (DGEBA) e a piperidina, modificado com poli(metacrilato de metila) (PMMA), foi estudado na faixa de temperatura de $60^{\circ} \mathrm{C}-120^{\circ} \mathrm{C}$. A concentração de PMMA e a temperatura de cura causam mudanças significativas na morfologia gerada. A massa molecular de PMMA provoca ligeiras mudanças para a observação da separação de fases e não afeta a velocidade da reação. O sistema modificado com PMMA mostra o efeito de retardação cinético e a velocidade de separação de fases é maior que a velocidade de polimerização.
\end{abstract}

Palavras-chave: Éter diglicidílico do bisfenol A (DGEBA), piperidina, poli(metacrilato de metila), separação de fases induzida por reação.

\section{Phase Separation Induced by Chemical Reaction in the System of Diglycidyl Ether of Bisphenol A and Piperidine with Poly(methyl methacrylate)}

Abstract: The phase separation and gelification behavior of epoxy system based on diglycidyl ether of bisphenol-A (DGEBA) and piperidine modified with poly(methyl methacrylate) (PMMA) were studied in the range between $60{ }^{\circ} \mathrm{C}$ and $120^{\circ} \mathrm{C}$. The morphology is influenced by the PMMA content in the blend and also by the cure temperature. The molecular weight of PMMA provokes slight changes on cloud point and does not affect the reaction rate. The systems modified by PMMA exhibited kinetic retardation effect but the cloud point rate was higher than the polymerization rate.

Keywords: Diglycidyl ether of bisphenol A (DGEBA), piperidine, poly(methyl methacrylate), reaction-induced phase separation.

\section{Introdução}

As resinas epoxídicas constituem uma das mais importantes classes de polímeros termorrígidos utilizados em adesivos, matrizes para compósitos reforçados e recobrimentos. Estes materiais apresentam excelentes propriedades mecânicas e estabilidade térmica, como resultado da natureza estrutural dos monômeros e da alta densidade de reticulação da matriz. No entanto, quando estes materiais são destinados a aplicações que solicitam alta resistência mecânica, é comum a incorporação de modificadores baseados na dispersão de uma segunda fase para a tenacificação da matriz. A dispersão da segunda fase pelo método de separação de fases induzida por reação química é de grande interesse para vários grupos de pesquisa ${ }^{[1-12]}$. A revisão mais recente relatada, que reúne diferentes aspectos teóricos e práticos relacionados à modificação de matrizes termorrígidas pelo método de separação de fases induzida por reação química, foi resultado da cooperação entre diferentes grupos ${ }^{[13]}$. Por este método é possível utilizar como modificadores tanto borrachas como termoplásticos, desde que sejam solúveis no sistema epoxídico e, durante a cura, ocorra a separação de fases.
A utilização de termoplásticos como modificadores de matrizes epoxídicas é bem conhecida. Em particular, o emprego de poli(metacrilato de metila) (PMMA), tanto na forma de homopolímero ${ }^{[14-20]}$, como de copolímeros graftizados $^{[21,22]}$ ou em bloco ${ }^{[23]}$, é de grande interesse, pois o PMMA é solúvel e não reage quimicamente com a resina epoxídica $^{[14,18,24]}$. Recentemente foi relatada, como alternativa para a obtenção de uma dispersão estável de partículas bifásicas do modificador na matriz epoxídica, a utilização de um copolímero graftizado constituído por um segmento não-solúvel e outro solúvel antes da reação quí$\mathrm{mica}^{[25]}$. Mais recentemente, foram relatados dois trabalhos onde são utilizados copolímeros tribloco constituídos por segmentos com solubilidades diferenciadas para propósitos de tenacificação ${ }^{[23,26]}$.

Neste trabalho estuda-se o sistema constituído pelo éter diglicidílico do bisfenol $\mathrm{A}$ e a piperidina com poli(metacrilato de metila) (PMMA), com o objetivo de obtenção de informações sobre o comportamento morfológico induzido por reação química. $\mathrm{O}$ efeito da temperatura de cura, concentração e massa molecular do PMMA na morfologia gerada, assim como a influência do modificador na velocidade da 
reação, foi investigada. O trabalho mostra a importância tanto do conhecimento da reação de cura do sistema epoxídico, quanto do estabelecimento da relação entre a separação de fases e a reação de cura, para uma correta compreensão da morfologia gerada por reação química.

\section{Experimental}

\section{Materiais}

A resina utilizada foi o éter diglicidílico do bisfenol A, (DGEBA, DER 331) com 191 g/eq., determinado por titulação e por uma metodologia relatada recentemente que utiliza a espectroscopia de ressonância magnética nuclear de prótons ${ }^{[27]}$. A resina epoxídica, antes de sua utilização, foi seca por tratamento a vácuo a $80{ }^{\circ} \mathrm{C}$, durante 24 horas. A piperidina (PIP, VETEC) foi purificada por destilação. Três polímeros de poli(metacrilato de metila) (PMMA), (1, 2, e 3$)$ com massas moleculares $\overline{\mathrm{Mn}}=(1,15 ; 2,41$ e 4,58$) \times 10^{4}$ e com polidispersão de 2,4;2,3 e 2,7, respectivamente, determinadas por cromatografia de exclusão por tamanho (SEC), foram sintetizados via radical livre por polimerização de metacrilato de metila em tolueno utilizando quantidades diferentes de 2,2'-Azo-bis-isobutironitrila (AIBN) como iniciador ${ }^{[28]}$.

\section{Preparação das misturas}

Sistemas binários de resina epoxídica com concentrações iguais a 3, 5, 7 e $10 \mathrm{phr}$ de PMMA (phr: partes de PMMA por cada 100 partes de resina), e com PMMA de diferentes massas moleculares foram preparados utilizando diclorometano como solvente. Após a solubilização dos componentes, o diclorometano foi removido sob vácuo, a $80^{\circ} \mathrm{C}$. Posteriormente, para a preparação dos sistemas modificados antes da cura, cada sistema binário foi tratado a vácuo, a $80^{\circ} \mathrm{C}$, durante 1 hora e resfriado à temperatura ambiente antes da adição de piperidina (PIP) (5 phr) (phr: partes de PIP por cada 100 partes de resina).

\section{Determinação do tempo de separação de fases e de gelificação}

O tempo de separação de fases e de gelificação foi determinado por experiências isotérmicas a diferentes temperaturas, de maneira visual, utilizando o termostato Haake W-26. Foi considerado que ocorre a separação de fases quando se observou o primeiro indício de turvação durante a experiência isotérmica. Considerou-se que ocorre a gelificação quando aparece o primeiro resíduo de material insolúvel em tetra-hidrofurano, como resultado de sucessivos ensaios de solubilidade a diferentes tempos para cada experiência isotérmica.

\section{Micrografias eletrônicas de varredura (SEM)}

Amostras de $50 \times 20 \times 2 \mathrm{~mm}$ foram preparadas por tratamento em estufa, pelo emprego de diferentes programas de cura, utilizando moldes de silicone. Após a cura, as amostras foram fraturadas à temperatura ambiente.
As superfícies de fratura das amostras cobertas com uma fina camada de ouro foram observadas utilizando microscópio Jeol-JMS-5300, operando a $20 \mathrm{kV}$. As micrografias foram analisadas utilizando o programa de imagens Globus que permitiu determinar os diâmetros das partículas e a posterior construção das curvas de distribuição de tamanhos. O diâmetro médio das partículas foi determinado utilizando a equação 1 .

$$
\text { diâmetro médio }=\frac{\sum \mathrm{nd}}{\sum \mathrm{n}}
$$

Onde $\mathrm{n}$ representa o número de partículas e $\mathrm{d}$ o diâmetro da partícula.

\section{Método calorimétrico}

Os sistemas modificados e o sistema epoxídico foram estudados por experiências isotérmicas (DSC) a $80^{\circ} \mathrm{C}$, utilizando o analisador Perkin-Elmer DSC-7, sob atmosfera de nitrogênio e quantidades de amostra na faixa de 15 a $20 \mathrm{mg}$.

\section{Resultados e Discussão}

\section{Influência do PMMA na velocidade da reação}

Para uma correta compreensão dos comportamentos do sistema epoxídico e do sistema modificado neste trabalho, é importante dizer que a piperidina é uma amina secundária, sendo bastante utilizada como agente de cura de resinas epoxídicas empregadas para formulações adesivas ${ }^{[29-35]}$. No sistema com piperidina, é característico que a temperatura de cura afete a temperatura de transição vítrea ${ }^{[29]} \mathrm{e}$ a energia de fraturas $^{[30-32]}$, como resultado da formação de diferentes estruturas de rede e/ou pela volatilização da piperidina. Segundo a literatura ${ }^{[33-35]}$, o mecanismo da reação deste sistema envolve duas etapas principais ilustradas na Figura 1. A primeira etapa consiste na reação de adição da piperidina com o grupo epoxídico (Figura 1, 1a), originando uma estrutura contendo as funções amina terciária e álcool, através de um mecanismo competitivo catalisado pelo grupo hidroxila e outro nãocatalisado, onde o primeiro predomina à baixa conversão. Este mecanismo é típico de sistemas que utilizam aminas primárias como agentes de cura ${ }^{[34,36]}$. A segunda etapa consiste na homopolimerização dos grupos epoxídicos iniciada pelo alcóxido correspondente à estrutura contendo os grupos amina terciária e álcool, a partir de um mecanismo aniônico, que compreende etapas de iniciação (Figura 1, 1b e 1c), propagação (Figura 1,2) e transferências (Figura 1, 3a e 3b), onde são formadas redes funcionalizadas (grupos vinílicos terminais), como resultado de transferências de cadeia que são favorecidas a altas temperaturas ${ }^{[33-35]}$. Esta segunda etapa corresponde ao mecanismo típico de sistemas que utilizam as aminas terciárias como agentes de cura ${ }^{[33]}$. Tal comportamento justifica que seja esperada uma dependência complexa do desempenho do sistema epoxídico com a temperatura. 


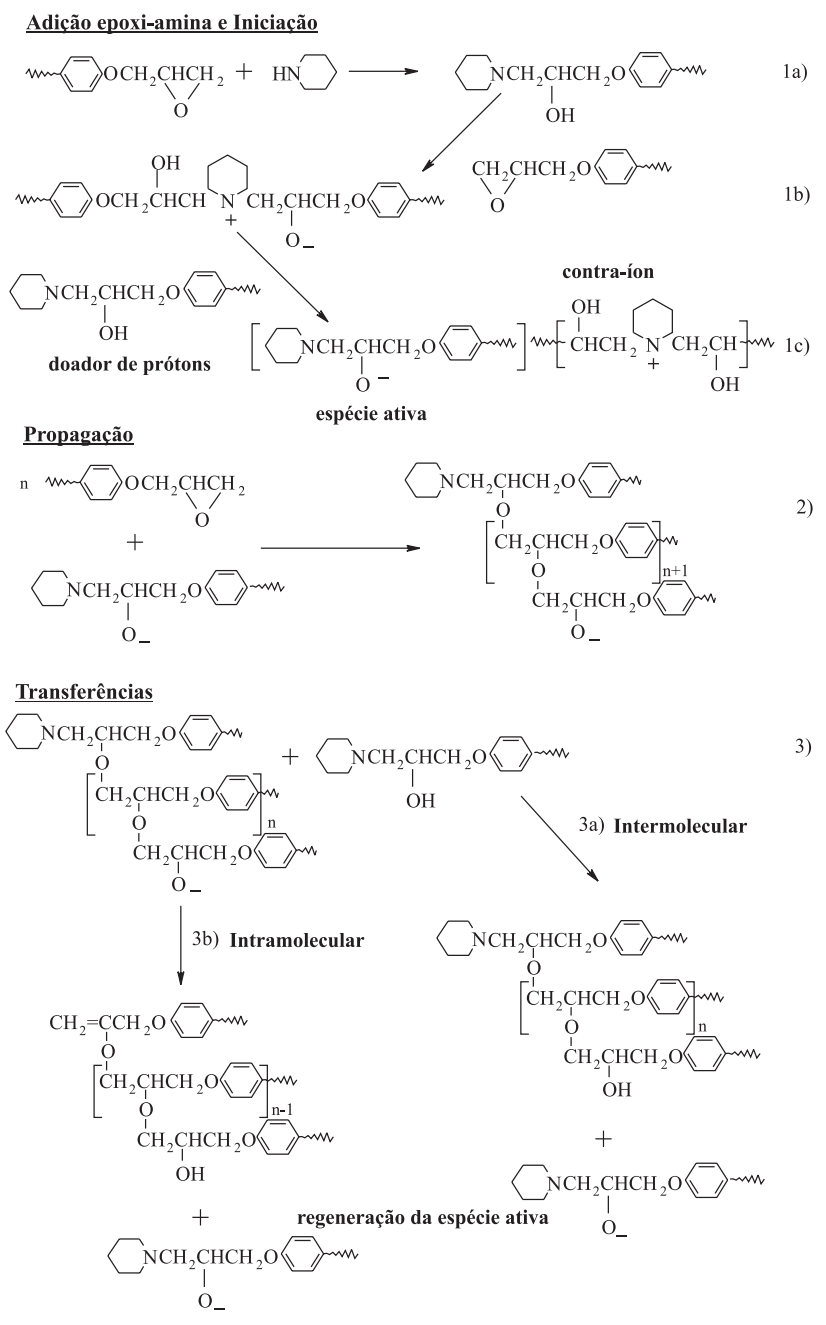

Figura 1. Mecanismo parcial da reação de cura do sistema epoxídico

A Figura 2 mostra os tempos de gelificação, determinados a diferentes temperaturas de cura para o sistema epoxídico e para os sistemas modificados com PMMA de diferentes massas moleculares. Os tempos de gelificação dos sistemas com a temperatura apresentaram dois comportamentos diferentes. Para baixas temperaturas $\left(60{ }^{\circ} \mathrm{C}\right.$ a $\left.80^{\circ} \mathrm{C}\right)$, o tempo de gelificação diminui com o aumento da temperatura, como era esperado. No entanto, acima de $80{ }^{\circ} \mathrm{C}$, aumenta com o aumento da temperatura. Isto pode ser devido à volatilização da piperidina a altas temperaturas ${ }^{[29,31,33]}$. Com isso, a concentração de estruturas contendo os grupos amina terciária e álcool diminui, diminuindo significativamente a velocidade da reação. Além disso, acredita-se que a conversão de gelificação aumente (aumenta o tempo de cura) com a elevação da temperatura, devido a uma possível diminuição da razão entre as velocidades de propagação e transferências. No entanto, a magnitude deste efeito sozinho não pode explicar o aumento tão significativo da gelificação acima de $80^{\circ} \mathrm{C}$, o que indica que a volatilização é o fator mais importante.

De modo comparativo, as medidas do tempo de gelificação entre os sistemas revelaram que a presença de PMMA provoca um pequeno efeito de retardação, considerando que a conversão de gelificação não muda pela presença do PMMA, já que este

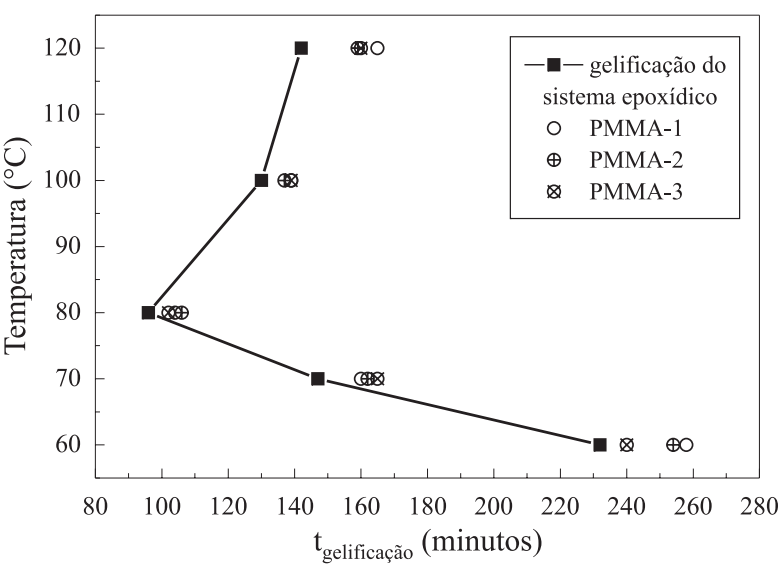

Figura 2. Tempo de gelificação determinado de maneira visual, a diferentes temperaturas, para o sistema epoxídico, e para três sistemas com 5 phr de PMMA de diferentes massas moleculares PMMA-1 $\left(\overline{\mathrm{Mn}}=1,15 \times 10^{4}\right)$, PMMA-2 $\left(\overline{\mathrm{Mn}}=2,41 \times 10^{4}\right)$ e PMMA-3 $\left(\overline{\mathrm{Mn}}=4,58 \times 10^{4}\right)$

modificador não participa da reação de cura ${ }^{[14,18,24]}$. Isto pode ser atribuído a que, antes da separação de fases, o modificador provoque uma diminuição relativa na concentração dos grupos funcionais (reação de adição do grupo epoxídico à piperidina), o que é denominado na literatura como efeito de diluição ${ }^{[6,18]}$. Além disto, a retardação pode estar relacionada à existência de mudanças na polaridade do meio reacional que afetem o período de indução para a formação da espécie ativa que inicia a polimerização aniônica (homopolimerização dos grupos epoxídicos). A massa molecular do PMMA parece não afetar a velocidade da polimerização apesar dos resultados mostrados na Figura 2, pois, para as diferentes temperaturas de cura utilizadas, não foram observadas diferenças significativas entre os tempos de gelificação.

Informação adicional da influência do PMMA na velocidade da reação foi obtida utilizando a calorimetria diferencial de varredura (DSC). A Figura 3 compara as curvas de conversão $v s$ tempo relativas aos dois sistemas modificados

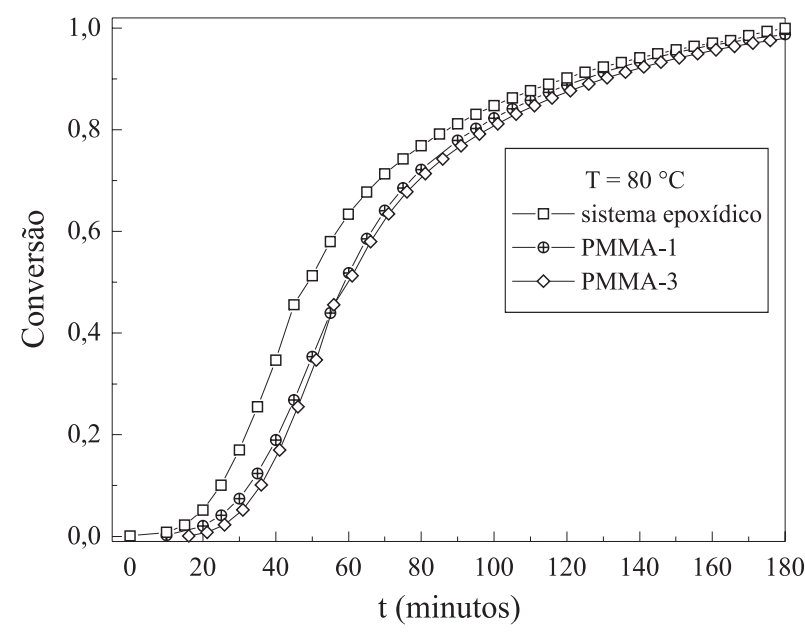

Figura 3. Curvas de conversão vs tempo obtidas por calorimetria diferencial de varredura (DSC), a partir de experiências a $80^{\circ} \mathrm{C}$, para o sistema epoxídico e para dois sistemas com $5 \mathrm{phr}$ de PMMA de diferentes massas moleculares PMMA-1 $\left(\overline{\mathrm{Mn}}=1,15 \times 10^{4}\right)$ e PMMA-3 $\left(\overline{\mathrm{Mn}}=4,58 \times 10^{4}\right)$ 
com aquela correspondente ao sistema epoxídico, obtidas por experiências de calorimetria isotérmica a $80^{\circ} \mathrm{C}$. A conversão foi obtida pela equação 2 .

$$
X=\frac{(\mathrm{dQ} / \mathrm{dt})}{\mathrm{Q}}
$$

Onde $\mathrm{X}$ representa a conversão no tempo t, dQ/dt calor parcial no tempo t, e $\mathrm{Q}$ calor total correspondente à curva calorimétrica

Como observado na Figura 3, a incorporação do PMMA no sistema provoca o efeito de retardação cinético e não ocorrem diferenças entre as velocidades de reação entre os dois sistemas modificados. Isto confirma que a massa molecular do modificador não afeta a velocidade da reação.

\section{Separação de fases e morfologia gerada}

Os três sistemas com PMMA de diferentes massas moleculares, preparados à temperatura ambiente, ficaram transparentes. No entanto, durante a realização das experiências isotérmicas a diferentes temperaturas tornaram-se opacos, o que revelou o fenômeno de separação de fases. A Figura 4 mostra de modo comparativo os tempos de separação de fases para dois sistemas modificados a diferentes temperaturas de cura, onde foi incluído o tempo de gelificação para fins comparativos.

A separação de fases ocorre em tempos próximos quando os sistemas modificados são comparados, mas este fenômeno sempre ocorre antes da gelificação. Este comportamento coincide com aquele relatado por outros pesquisadores ${ }^{[14,16-18]}$, no estudo de sistemas que utilizam, como agentes de cura, aminas primárias e anidrido de ácido, com PMMA de massas moleculares semelhantes às utilizadas neste trabalho. Além disto, a separação de fases apresenta uma ligeira e sistemática diminuição, com o aumento da massa molecular do modificador. Para o sistema com PMMA-3, que corresponde

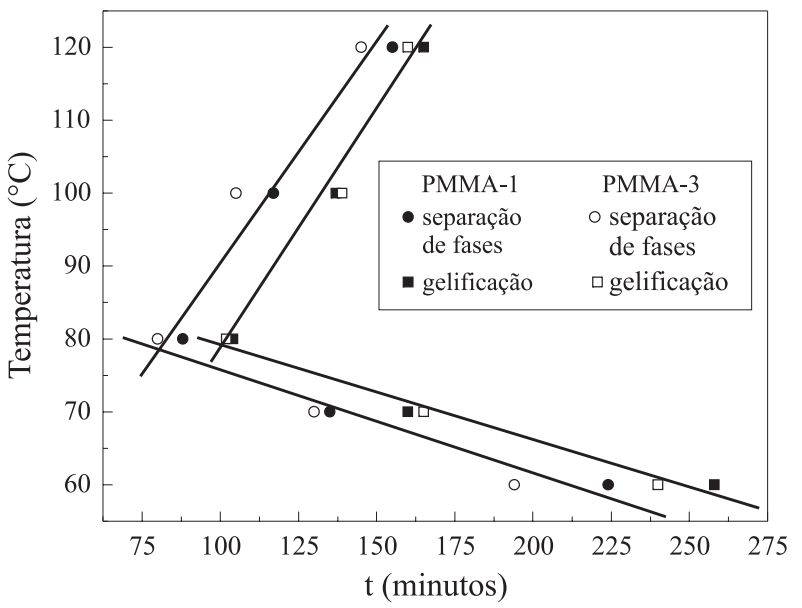

Figura 4. Tempo de separação de fases determinado de maneira visual, a diferentes temperaturas, para três sistemas com 5 phr de PMMA de diferentes massas moleculares PMMA-1 $\left(\overline{\mathrm{Mn}}=1,15 \times 10^{4}\right)$, PMMA-2 $\left(\overline{\mathrm{Mn}}=2,41 \times 10^{4}\right)$ e PMMA-3 $\left(\overline{\mathrm{Mn}}=4,58 \times 10^{4}\right)$ ao modificador de maior massa molecular, a separação de fases é alcançada em menor tempo. Este comportamento indica que o aumento da massa molecular do modificador conduz à separação de fases a uma conversão mais baixa. Esta afirmação está fundamentada no resultado encontrado neste trabalho, onde a massa molecular do PMMA não afeta a velocidade da reação. Este resultado coincide com o relatado por Williams et al. ${ }^{[13,37,38]}$ no modelo que utiliza a equação de Flory-Huggins para descrever o fenômeno de separação de fases induzido por reação química.

A Figura 5 mostra as micrografias eletrônicas de varredura (SEM) correspondentes à morfologia gerada após a cura a $80{ }^{\circ} \mathrm{C}$, durante 4 horas, para os sistemas com 3, 5, 7 e 10 phr de PMMA-1 $\left(\overline{\mathrm{Mn}}=1,15 \times 10^{4}\right)$. Neste caso, o tempo de cura garante que o sistema esteja a uma conversão superior à gelificação, o que assegura que a morfologia gerada não seja modificada ${ }^{[1,29]}$. Para as concentrações utilizadas, a morfologia gerada é bifásica do tipo fase dispersa/matriz, onde aparece uma fase contínua com regiões estriadas, que inclui também regiões lisas, e outra fase dispersa de cor branca na forma de partículas esféricas. As partículas esféricas de cor branca foram atribuídas à fase de PMMA, pela observação de mudança dos diâmetros com a temperatura de cura. A existência de vazios indica que existe uma fraca interação entre as partículas e a matriz.

Nas micrografias da Figura 5, observa-se que os tamanhos das partículas dispersas da fase de PMMA aumentam com o aumento da concentração do modificador. Para as concentrações de 3 phr a 10 phr, o diâmetro médio varia de 0,31 a 0,64 $\mathrm{mm}$. Este comportamento sugere que, nesta faixa de concentrações, o aumento da concentração de PMMA conduza à
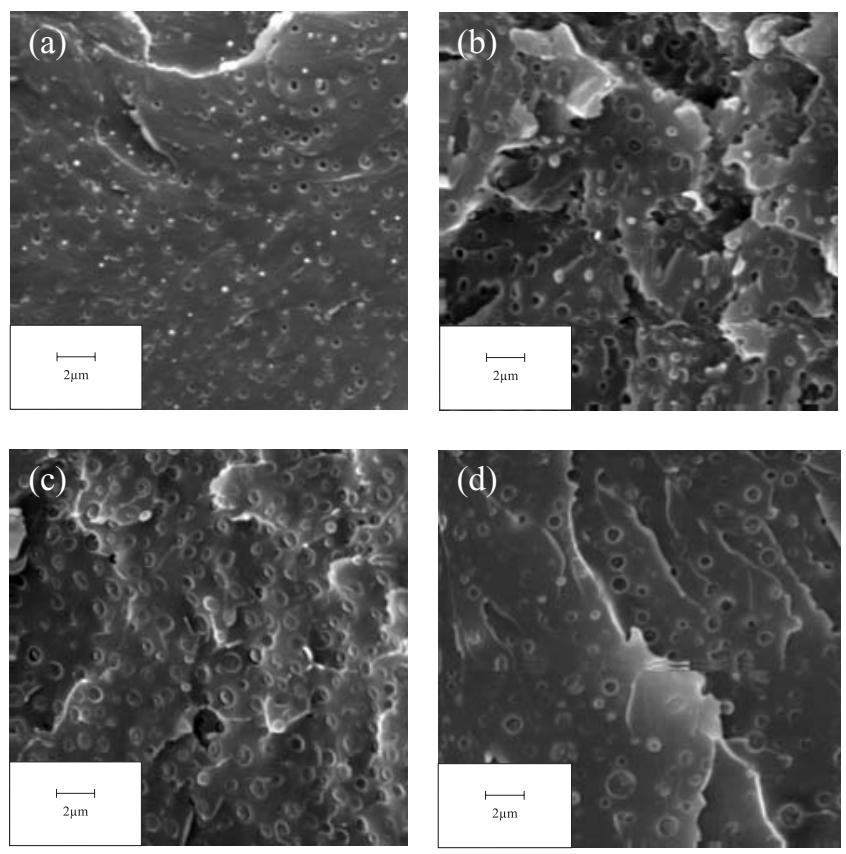

Figura 5. Micrografias eletrônicas (SEM) da morfologia gerada para os sistemas com PMMA-1 $\left(\overline{\mathrm{Mn}}=1,15 \times 10^{4}\right)$, após a cura a $80^{\circ} \mathrm{C}$, durante 4 horas, utilizando diferentes concentrações de PMMA. a) $3 \mathrm{phr}$, b) $5 \mathrm{phr}$, c) $7 \mathrm{phr}$ e d) $10 \mathrm{phr}$ 

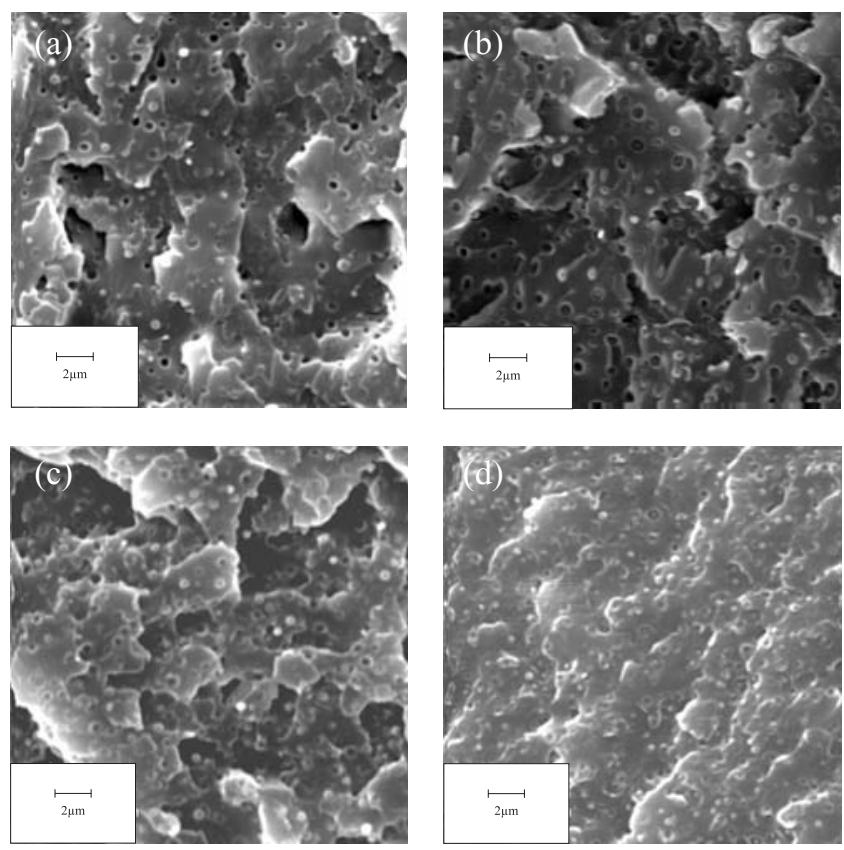

Figura 6. Micrografias eletrônicas (SEM) da morfologia gerada pelo sistema com 5 phr de PMMA-1 ( $\left.\overline{\mathrm{Mn}}=1,15 \times 10^{4}\right)$ após cura isotérmica a diferentes temperaturas. a) $60^{\circ} \mathrm{C}$, durante 6 horas, b, c, e d) $80{ }^{\circ} \mathrm{C}, 100^{\circ} \mathrm{C}$, e $120^{\circ} \mathrm{C}$, por 4 horas, respectivamente

separação de fases a uma conversão mais baixa (menor tempo), considerando que esse aumento não afete a velocidade da reação. Isto sugere também que, com o aumento da concentração de modificador, a separação de fases ocorra a uma viscosidade menor, o que favorece o crescimento das partículas. Estas considerações e o comportamento experimental observado para os domínios de PMMA coincidem com o relatado para outros sistemas epoxídicos modificados, tanto com borrachas como com termoplásticos ${ }^{[1,8-9,11,13,20,26,30]}$.

A influência da temperatura de cura para o sistema com 5 phr de PMMA-1 ( $\left.\overline{\mathrm{Mn}}=1,15 \times 10^{4}\right)$ é apresentada na Figura 6, onde são mostradas as micrografias eletrônicas (SEM) correspondentes à morfologia gerada depois da cura isotérmica a diferentes temperaturas $\left(60^{\circ} \mathrm{C}\right.$ durante 6 horas, e $80^{\circ} \mathrm{C}$, $100^{\circ} \mathrm{C}$ e $120^{\circ} \mathrm{C}$ durante 4 horas). Por motivos práticos foram utilizados tempos de cura reduzidos, de maneira que a morfologia gerada ficasse congelada pela gelificação. Neste caso, para uma melhor observação do comportamento morfológico, foram construídas as curvas de distribuição de tamanho. A Figura 7 mostra o comportamento morfológico representado como uma função de distribuição do tamanho de partículas para as diferentes temperaturas utilizadas.

$\mathrm{Na}$ Figura 7 é evidente que, para baixas temperaturas $\left(60{ }^{\circ} \mathrm{C}\right.$ a $\left.80{ }^{\circ} \mathrm{C}\right)$, o incremento da temperatura provoca o deslocamento discreto da curva de distribuição para maiores tamanhos e, para altas temperaturas $\left(100{ }^{\circ} \mathrm{C}\right.$ a $\left.120^{\circ} \mathrm{C}\right)$, ocorre o contrário. De acordo com a literatura, o comportamento morfológico, onde o aumento da temperatura de cura acarreta em um aumento do tamanho dos domínios até alcançar um valor máximo, corresponde a sistemas onde a velocidade da separação de fases é mais rápida que a velocidade de

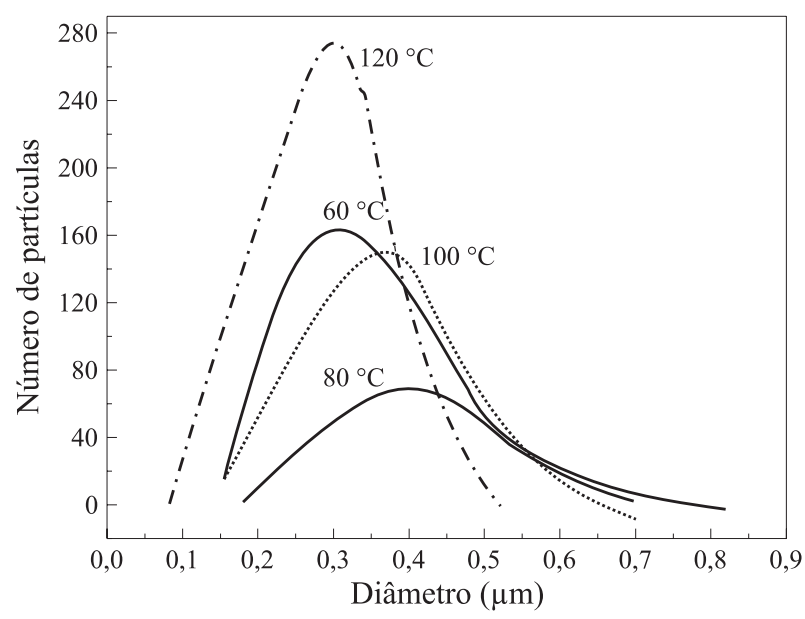

Figura 7. Influência da temperatura na curva de distribuição do tamanho de partículas para o sistema com 5 phr de PMMA-1 $\left(\overline{\mathrm{Mn}}=1,15 \times 10^{4}\right)$. Cura isotérmica a $60{ }^{\circ} \mathrm{C}$, durante 6 horas, e a $80^{\circ} \mathrm{C}, 100^{\circ} \mathrm{C}$ e $120^{\circ} \mathrm{C}$, durante 4 horas

reação ${ }^{[1,13,30]}$. Isto sugere que neste sistema seja possível o controle da separação de fases a partir de mudanças na velocidade de polimerização ${ }^{[13]}$. Isto significa que, com o aumento da velocidade de polimerização, pode não ser observada a separação de fases macroscópica, como resultado do aumento na velocidade de reação, o que provoca uma diminuição significativa tanto da fração volumétrica da fase dispersa, quanto dos tamanhos de partículas. Neste sistema a velocidade de polimerização máxima pode ser alcançada pelo aumento da temperatura de cura, mantendo constante a concentração de piperidina. Isto será tratado posteriormente neste trabalho.

Para explicar o comportamento morfológico em função de temperatura de cura, é necessário relacionar o máximo das curvas de distribuição à viscosidade do meio reacional, no momento onde a separação de fases ocorre, e considerar que o mecanismo de nucleação e crescimento seja predominante. Para baixas temperaturas $\left(60^{\circ} \mathrm{C}\right.$ a $\left.80^{\circ} \mathrm{C}\right)$, é possível aceitar que o aumento da temperatura acarreta na diminuição da viscosidade. Entretanto, o aumento da temperatura também resulta na separação de fases em menor tempo (conversão mais baixa), o que favorece o crescimento das partículas e conseqüentemente o número de partículas da fase de PMMA diminui. Além disto, a diminuição da viscosidade como conseqüência do aumento da temperatura de cura, provoca o aumento do coeficiente de difusão que conduz ao aumento dos diâmetros de partículas como tem sido relatado ${ }^{[13,37]}$.

Para altas temperaturas $\left(100^{\circ} \mathrm{C}\right.$ a $\left.120^{\circ} \mathrm{C}\right)$, é necessário considerar que a solubilidade do modificador seja de temperatura superior crítica (UCST), de maneira que o aumento da temperatura aumente a solubilidade do PMMA. Este efeito faz com que a separação de fases seja retardada, ou seja, que ocorra a um tempo de reação maior, que corresponde a uma conversão mais elevada. Isto significa que o aumento da solubilidade do modificador faz com que a separação de fases aconteça em um meio de maior viscosidade, o que favorece a formação de um maior número de partículas de menores tamanhos, como tem sido antecipado na literatura utilizando 

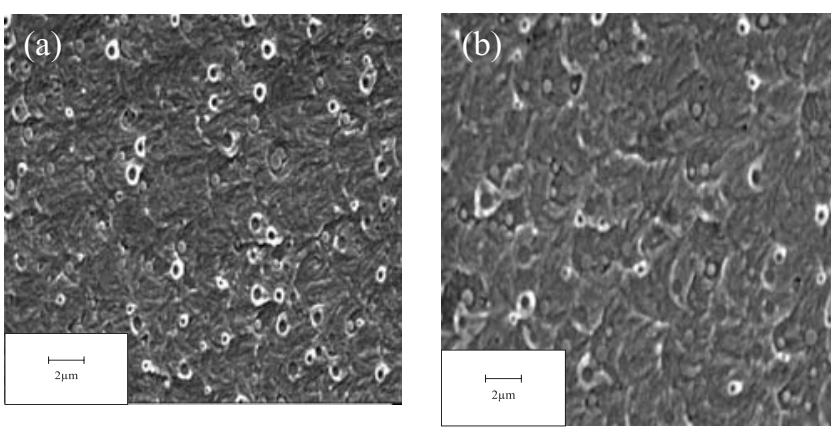

Figura 8. Micrografias eletrônicas (SEM) da morfologia gerada pelo sistema com $5 \mathrm{phr}$ de PMMĀ-1 $\left(\mathrm{Mn}=1,15 \times 10^{4}\right)$, após o programa de cura, a $60{ }^{\circ} \mathrm{C}$, durante 30 minutos, e depois por 4 horas, (a) $100^{\circ} \mathrm{C}$, e (b) $120{ }^{\circ} \mathrm{C}$

o modelo de separação de fases ${ }^{[37,38]}$ e comprovado também por Verchere et al. ${ }^{[1]}$.

A Figura 8 mostra as micrografias eletrônicas (SEM) correspondentes à morfologia gerada por dois sistemas com 5 phr de PMMA-1 $\left(\overline{\mathrm{Mn}}=1,15 \times 10^{4}\right)$ depois de um programa de cura em duas etapas. Na etapa inicial, a $60{ }^{\circ} \mathrm{C}$, durante 30 minutos, para os dois sistemas e depois a $100^{\circ} \mathrm{C}$ ou $120^{\circ} \mathrm{C}$, por 4 horas, para cada sistema de modo independente. Neste caso, o tratamento inicial a $60^{\circ} \mathrm{C}$ assegura que toda a piperidina se adicione ao grupo epoxídico, formando estruturas com os grupos amina terciária e álcool, o que garante a máxima concentração de iniciador para a polimerização aniônica (homopolimerização). Desta forma, espera-se que a velocidade de polimerização a $120{ }^{\circ} \mathrm{C}$ seja máxima, e que o programa de cura não comprometa as propriedades mecânicas do sistema epoxídico ${ }^{[30-33]}$. O objetivo é alcançar mudanças na morfologia visando propriedades diferenciadas ${ }^{[15,17,19,20]}$. De $\approx 100^{\circ} \mathrm{C}$ a $120^{\circ} \mathrm{C}$, o diâmetro médio dos domínios diminui de 0,32 a 0,26 $\mu \mathrm{m}$. Este resultado confirma que neste sistema a velocidade de separação de fases é maior que a velocidade de polimerização. Além disto, a máxima velocidade de polimerização não parece afetar de modo significativo o tamanho de partículas, o que indica que a velocidade de separação de fases é bem maior que a velocidade de reação. Isto justifica que neste sistema, o controle da separação de fases pelo aumento da velocidade de reação fica limitado.

\section{Conclusões}

Na modificação do sistema epoxídico com poli(metacrilato de metila) (PMMA), a concentração de modificador e a temperatura de cura afetam de modo significativo a morfologia gerada. $\mathrm{O}$ aumento da concentração de PMMA provoca o aumento de tamanho de partículas. Na faixa de $60{ }^{\circ} \mathrm{C}$ a $80{ }^{\circ} \mathrm{C}$, o incremento da temperatura provoca $\mathrm{o}$ aumento de tamanho dos domínios e para a faixa de $100{ }^{\circ} \mathrm{C}$ a $120^{\circ} \mathrm{C}$, ocorre o contrário. $\mathrm{O}$ aumento da massa molecular do PMMA conduz à diminuição do tempo de separação de fases e não afeta a velocidade de polimerização. O PMMA causa o efeito de retardação cinético e neste sistema a velocidade da separação de fases é bem maior que a velocidade de reação, o que limita o controle da separação de fases pelo aumento da velocidade de polimerização.

\section{Agradecimentos}

Os autores agradecem à CAPES, CNPq, PADCT e FAPERJ pelo apoio financeiro recebido e as oportunas sugestões realizadas pelo professor Roberto J.J. Williams.

\section{Referências Bibliográficas}

1. Verchere, D.; Pascault, J.P.; Sautereau, H.; Moschiar, S.M.; Riccardi, C.C. \& Williams, R.J.J. - J. Appl. Polym. Sci., 42, p.701 (1991).

2. Girard-Reydet, E.; Sautereau, H.; Pascault, J.P.; Keates, P.; Narvard, P.; Thollet, G. \& Vigier, G. - Polymer, 39, p.2269 (1998).

3. Alig, I.; Jenninger, W. \& Schawe, J.E.K. - Thermochimica Acta, 330, p.175 (1999).

4. Swier, S. \& Van Mele, B. - Thermochimica Acta, 330, p. 175 (1999).

5. Zhang, J.; Zhang, H. \& Yang, Y. - J. Appl. Polym. Sci., 72, p.59 (1999).

6. Martinez, I.; Martin, M.D.; Eceiza, A.; Oyanguren, P. \& Mondragon, I. - Polymer, 41, p.1027 (2000).

7. Wise, C.W.; Cook, W.D. \& Goodwin, A.A. - Polymer, 41, p.4625 (2000).

8. Ratna, D. \& Simon, G.P. - Polymer, 42, p.7739 (2001).

9. Kaynak, C.; Ozturk, A. \& Tincer, T. - Polym. Int., 51, p.749 (2002).

10. Zheng, Q.; Kuitian Tan, H.; Peng, M. \& Pan, Y. - J. Appl. Polym. Sci., 85, p.950 (2002).

11. López, J.; Ramírez, C.; Abad, M.J.; Barral, L.; Cano, J. \& Díez, J. - J. Appl. Polym. Sci., 85, p.1277 (2002).

12. Chikhi, N.; Fellahi, S. \& Bakar, M. - Eur. Polym. J., 38, p.251 (2002).

13. Williams, R.J.J.; Rozenberg, B.A. \& Pascault, J.P. - Adv. Polym. Sci., 128, p.95 (1997).

14. Gómez, C.M. \& Bucknall, C.B. - Polymer, 34, p.2111 (1993).

15. Mondragon, I.; Remiro, P.M.; Martin, M.D.; Valea, A.; Franco, M. \& Bellenguer V. - Polym. Int., 47, p.152 (1998).

16. Galante, M.J.; Oyanguren, P.A.; Andromaque, K.; Frontini, P.M. \& Williams, R.J.J. - Polym. Int., 48, p.642 (1999).

17. Remiro, P.M.; Riccardi, C.C.; Corcuera, M.A. \& Mondragon, I. - J. Appl. Polym. Sci., 74, p.772 (1999).

18. Ritzenthaler, S.; Girard-Reydet, E. \& Pascault, J.P. Polymer, 41, p.6375 (2000). 
19. Remiro, P.M.; Marieta, C.; Riccardi, C.C. \& Mondragon, I. - Polymer, 42, p.9909 (2001).

20. Stefani, P.M.; Riccardi, C.C.; Remiro, P.M. \& Mondragon, I. - Polym. Ing. Sci. 41, p.2013 (2001).

21. Rezaifard, A.H.; Hodd, K.A.; Tod, D.A. \& Barton, J.M. Int. J. Adhesion and Adhesives, 14, p.153 (1994).

22. Ochi, M. \& Shinzi, S. - Polymer, 40, p.1667 (1999).

23. Ritzenthaler, S.; Court, F.; David, L.; Girard-Reydet, E.; Leibler, L. \& Pascault, J.P. - Macromolecules, 35, p.3245 (2002).

24. Woo, E.M. \& Wu, M.N. - Polymer, 37, p.2485 (1996).

25. González, F.; Soares, B.G. \& Williams, R.J.J. - Polym. Int., 51, p.1340 (2002).

26. Ritzenthaler, S.; Court, F.; Girard-Reydet, E.; Leibler, L. \& Pascault, J.P. - Macromolecules, 36, p.118 (2003).

27. González, F. \& Soares, B.G. - Polymer Testing, 22, p.51 (2003).

28. González F. - "Resinas Epoxídicas Modificadas com Polímeros Termoplásticos", Tese de Doutorado, Universidade Federal do Rio de Janeiro, Brasil (2001).

29. Manzione, L.T.; Gillham, J.K. \& McPherson, C.A. - J. Appl. Polym. Sci., 26, p.889 (1981).
30. Butta, E.; Levita, G.; Marchetti, A. \& Lazzeri, A. - Polym. Ing. Sci., 26, p.63 (1986).

31. Kinloch, A.J.; Finch, C.A. \& Hashemi, S. - Polym. Com., 28, p.322 (1987).

32. Shaw, S.L. \& Tod, D.A. - J. Adhesion, 28, p.231 (1989).

33. Cuadrado, T.R.; Almaraz, A. \& Williams, R.J.J. - "Curing of Epoxy Resins with Piperidine", in: Crosslinked Epoxies, (Ed. B. Sedlácek J. Kahovec); De Gruyter, Berlin, p.170 (1978)

34. Galego, N.; Vázquez, A. \& Williams, R.J.J. - Polymer, 35, p.857 (1994).

35. González, F.; Ortiz, P. \& Galego, N. - Polym. Int., 42, p.163 (1997).

36. González, F. \& Galego, N. - Polímeros: Ciência e Tecnologia, 10, p. 218 (2000).

37. Williams, R.J.J.; Borrajo, J.; Adabbo, H.E. \& Rojas, A.J. - “A Model for Phase Separation During a Thermoset Polymerization", in: Rubber-Modified Thermoset Resin, Adv. Chem. Ser. 208, (Ed. Riew K. and Gillham J.K.); American Chemical Society, Washington DC, p.195(1984).

38. Vázquez, A.; Rojas, A.J.; Adabbo, H.E.; Borrajo, J. \& Williams, R.J.J. - Polymer, 28, p.1156 (1987). 